\title{
Acceptance and commitment therapy as a web-based intervention for depressive symptoms: randomised controlled trial
}

Wendy T. M. Pots, Martine Fledderus, Peter A. M. Meulenbeek, Peter M. ten Klooster, Karlein M. G. Schreurs and Ernst T. Bohlmeijer

\section{Background}

Depression is a highly prevalent disorder, causing a large burden of disease and substantial economic costs. Webbased self-help interventions seem promising in promoting mental health

\section{Aims \\ To compare the efficacy of a guided web-based intervention based on acceptance and commitment therapy (ACT) with an active control (expressive writing) and a waiting-list control condition (Netherlands Trial Register NTR1296). \\ Method \\ Adults with depressive symptoms from the general population were randomised to ACT $(n=82)$, expressive writing $(n=67)$ or waiting-list control $(n=87)$. The main outcome was reduction in depressive symptoms assessed with the Center for Epidemiological Studies - Depression scale.}

\section{Results}

Significant reductions in depressive symptoms were found following the ACT intervention, compared with the control group (Cohen's $d=0.56$ ) and the expressive writing intervention $(d=0.36)$. The effects were sustained at 6-month and 12-month follow-up.

\section{Conclusions}

Acceptance and commitment therapy as a web-based public mental health intervention for adults with depressive symptoms can be effective and applicable.

\section{Declaration of interest}

None.

\section{Copyright and usage}

(c) The Royal College of Psychiatrists 2016.
Depression is a highly prevalent disorder with a large impact on quality of life and high economic costs. ${ }^{1}$ The 12 -month prevalence of a depressive disorder in The Netherlands is estimated at 5.6\% for ages 18-64 years. ${ }^{2}$ The most important risk factor for developing major depressive disorder is the presence of clinically relevant depressive symptoms. ${ }^{3}$ Public mental health interventions targeting people with such symptoms are a promising strategy to reduce the prevalence of depression. These interventions can be successful, ${ }^{1}$ but recruitment is a challenge. ${ }^{4}$ Web-based interventions provide an opportunity to overcome this challenge by tackling the reasons for low participation rates, such as stigma associated with mental disorders or restrictions in time. ${ }^{4,5}$ Meta-analyses have shown that web-based interventions based on cognitive-behavioural therapy (CBT) are effective for the prevention of full-blown depression. ${ }^{6,7}$ One specific CBT intervention is acceptance and commitment therapy (ACT) $;^{8}$ this is an evidence-based treatment that focuses on promoting psychological flexibility, defined as the ability to act effectively in accordance with personal values even in the presence of life adversities. ${ }^{9}$ Despite the efficacy of ACT in various self-help formats, ${ }^{10}$ its efficacy as a web-based self-help intervention for adults with depressive symptoms or mild depressive disorder has not been studied. Our aim was to examine the effects of such an intervention in a large randomised controlled trial. This is the first study to compare this therapy not only with a waiting-list control but also with an active control condition based on expressive writing, ${ }^{11}$ and to evaluate its outcomes up to a year later.

\section{Method}

The study was approved by an independent medical ethics committee for research in mental health settings in The
Netherlands (METiGG; NL33619.097.100) and recorded in The Netherlands Trial Register (NTR1296). It is a pragmatic, randomised controlled trial with three arms: the ACT intervention, an active control condition (expressive writing) and a waiting-list control condition. Based on previous results randomisation was stratified according to gender, education (low $v$. middle-high) and age ( $\leqslant 50 v .>50$ years), ${ }^{12,13}$ using a computer-generated list that was concealed from the investigators. A sample size of 50 participants per condition was needed to detect an effect size of 0.40 (Cohen's $d$ ) for the primary outcome, ${ }^{12,13}$ with a statistical power of $(1-\beta)=0.80$ in a twotailed test $(P<0.05)$. Taking into account a drop-out rate of $40 \%$ (for web-based interventions), ${ }^{14} 235$ people were needed for randomisation. At 6 months after baseline those in the waiting-list control group received either the ACT intervention or the expressive writing intervention, which were offered as a choice. The assessment points were at baseline $\left(T_{0}\right)$, posttreatment 3 months after baseline $\left(T_{1}\right)$ and at 6 months $\left(T_{2}\right)$ and 12 months $\left(T_{3}\right)$ after baseline. Only participants in the intervention conditions received a follow-up measurement at $T_{3}$, since the waiting-list group received their intervention after $T_{2}$ and were excluded from later analyses.

\section{Participants and procedure}

Participants were recruited in January and February 2011 through advertisements in Dutch national newspapers and on the internet, asking for participation in research on coping with negative emotions through the use of a free web-based intervention. A webpage created for the purpose of this study included an outline of the study design and an option to register for participation in the trial. Within the webpage candidates filled out a secured computerised informed consent form. After informed consent 
had been received, initial screening was conducted online for checking the inclusion and exclusion criteria by use of a self-report questionnaire in a fully automated computerised assessment battery. Individuals were invited to participate if they met the following inclusion criteria: an age of 18 years or older with mild to moderate depressive symptoms, defined as a score above 10 on the Dutch version of the Center for Epidemiological Studies Depression (CES-D) scale, ${ }^{12}$ and completion of the baseline measurement $\left(T_{0}\right)$. Applicants were excluded if they reported few depressive symptoms ( $\leqslant 10$ on the CES-D), had received psychological or psychopharmacological treatment for a mental complaint within the past 3 months, had reading or writing problems due to insufficient Dutch language skills or were unable to invest approximately $30 \mathrm{~min}$ per day up to $3 \mathrm{~h}$ per week in the intervention and daily practice. Furthermore, applicants were excluded if diagnosed with a current severe mental disorder or had a moderate to high suicide risk according to the Dutch version of the Mini International Neuropsychiatric Interview (MINI) and the Sheehan Disability Scale (SDS). ${ }^{15-17}$ These individuals were excluded because they would require more intensive treatment, and were advised to consult their general practitioner. After passing the initial screening procedure all participants were contacted by telephone for a semi-structured interview using the MINI and the SDS. Together with the initial screening this constituted the baseline assessment. Five Master's degree students in psychology conducted the telephone interviews. They attended a 1-day workshop and were supervised during the assessments by a licensed clinical psychologist (W.T.M.P.). Decisions on participation in the study, based on the initial screening and the interview, were provided to the candidates at the end of the clinical interview with further information on participation or, when excluded, information on how to obtain adequate care. All approved participants received an email with instructions on how and when to log into the system.

All clinical interviewers were masked to the randomised condition. Participants were randomised after the clinical interview, and at $T_{2}$ the contact information needed for the clinical interview was held separately from the results of the randomisation procedure. Additionally, all interviewers were instructed to state explicitly at the start of the interview that they were unaware of which condition the participants were randomised to and that this masking was needed to ensure the interview was conducted in a non-biased manner.

\section{Interventions}

\section{Acceptance and commitment therapy}

The web-based ACT intervention was based on a self-help intervention, 'Living to the Full.' ${ }^{18}$ This has shown to be effective in promoting psychological flexibility, both as a group course and as a self-help intervention with email support. ${ }^{12,13}$ People who are psychologically flexible also score highly on acceptance, which is seen as a more effective strategy for regulating negative emotions and thoughts than experiential avoidance, i.e. persistent and generally fruitless attempts to avoid unwanted private experiences such as feelings, thoughts and bodily sensations. ${ }^{19,20}$ The web-based intervention comprised nine online modules, divided into three parts. The modules were based on six core processes of ACT that together promote psychological flexibility. These core processes are acceptance (active and aware embracing of aversive internal experiences); cognitive defusion (creating a context in which undesirable functions of thoughts disappear); contact with the present moment (mindfulness); self as context (the sense of oneself as the observer of one's thoughts, feelings and experiences); values (choosing values in different life domains); and committed action (commitment to choices on the basis of these values). ${ }^{9}$ In the first part of the intervention participants reflect on their avoidance and control strategies and whether these are effective in the long run. In the second part participants learn how to stay in contact with their present experiences without trying to avoid or control them. Cognitive defusion and experiencing self as context are practised. In the third part the focus is on becoming aware of one's most important personal values and making decisions based on these values. An additional focus is relapse prevention, which includes selfmanagement and action plans. Each module uses experiential exercises and metaphors to illustrate the ACT process, as well as text messages, tailored stories for motivation and an option to personalise the homepage. Furthermore, participants were encouraged to practise daily mindfulness exercises, designed to reduce stress. ${ }^{21}$ These exercises lasted on average $10-15 \mathrm{~min}$ and were provided on audio, downloadable within the web-based intervention. For a comprehensive description of the development of the intervention, see Kelders et al. ${ }^{22}$

\section{Active control condition}

The active control condition was a web-based intervention based on Pennebaker's expressive writing paradigm, ${ }^{11}$ which has shown small effects on various mental health outcomes. ${ }^{23}$ Expressive writing generally involves asking participants to write about a highly stressful experience (particularly their deepest thoughts and feelings), usually in three or four sessions. We extended and adapted Pennebaker's method into a web-based format equivalent to that of the ACT intervention, comprising nine online sessions presented in three parts. Each session started with a psychoeducational paragraph on emotions and emotion regulation, followed by instructions in the expressive writing method. This consisted of writing about emotional experiences for $15-30 \mathrm{~min}$ on at least 3 days a week. The first three sessions focused on expressive writing about negative experiences. In sessions four to six participants looked back at their experiences with expressive writing in the first part, and focused on emotion regulation and reappraisal of emotions; these modules were based on Gross's process model of emotion regulation, ${ }^{24}$ and were added to extend the intervention to 9 weeks. In the last three modules of the intervention participants focused on writing about positive experiences and self-management for preventive purposes.

\section{Waiting-list control}

Participants in the waiting-list control group were offered no intervention but were free to access other forms of care (as were all participants once they were eligible). They were instructed that, should they encounter symptomatic deterioration or other difficulties over the course of the study, they were to seek help from their family, general practitioner or other sources, as they normally would. Six months after baseline these participants could start a web-based intervention of their choice. Based on the preliminary results of this study at $T_{2}$, the participants were advised to start with ACT.

\section{Counselling}

Participants in the web-based ACT and expressive writing interventions were instructed to complete one session per week, and had 12 weeks in total to complete the nine sessions. After completing a session, participants wrote an email to their counsellor reflecting on the process and using the opportunity to ask questions. They could proceed to the next session after receiving personal feedback from their counsellor. The feedback 
messages of the counsellors contained the key learning points and goals of the completed session, feedback on the key exercises and a preview of the following session. The feedback was accompanied by positive and encouraging support. Participants received automatic email messages when they completed a session, when personal feedback was received, and to remind them to finish a session or to start a new session. A session was fulfilled when all exercises were completed.

Five psychology Master's degree students provided the email support in both the experimental and the active control conditions. They attended a 2-day workshop from licensed doctors of clinical psychology with ample experience in CBT, ACT and expressive writing (K.M.G.S. and W.T.M.P.), in which they studied the web-based interventions and practised writing emails in the roles of both client and counsellor. Each counsellor provided personal feedback to 25-30 participants during the intervention, supervised by a clinical psychologist. The counsellors were given a total of $3 \mathrm{~h}$ on the counselling of an individual participant.

\section{Assessment}

The primary outcome measure was depressive symptoms measured by the Dutch version of the CES-D (20 items, total score $0-60)$. Higher scores mean more depressive symptoms. ${ }^{25,26}$ Secondary outcome measures were diagnostic classification, anxiety symptoms, positive mental health, psychological flexibility and mindfulness. The diagnostic classification was assessed with the MINI, ${ }^{15,16}$ supplemented with the SDS to measure the severity of the disorder. ${ }^{17}$ Severity was defined as at least two areas of functioning with severe role impairment due to the disorder according to the SDS. The MINI and SDS assessments were conducted by telephone at $T_{0}$ and $T_{2}$. Anxiety was measured with the Hospital Anxiety and Depression Scale - Anxiety subscale (HADS-A; 7 items, total score 0-21). Higher scores mean more anxiety symptoms. ${ }^{27,28}$ Positive mental health was measured with the Mental Health Continuum - Short Form (MHC-SF; 14 items, each scored $0-5$ ), which measures three dimensions of positive mental health: emotional, social and psychological well-being. ${ }^{29}$ In this study the total MHC-SF score was used, with higher scores indicating greater emotional, social and psychological well-being. ${ }^{29,30}$ The Acceptance and Action Questionnaire II (AAQ-II; 10 items, total score 10-70) was used to measure the participants' willingness to be in contact with negative private events, their acceptance of these events and the ability to live according to their values. Higher scores indicate greater psychological flexibility. ${ }^{31,32}$ The Five Facet Mindfulness Questionnaire - Short Form (FFMQ-SF; 24 items, total score 24-120) was used to measure mindfulness in five dimensions: observing, describing, acting with awareness, non-judging and non-reactivity. Facet scores range from 5 to 25 (except for observing, which ranges from 4 to 20), with higher scores indicating greater mindfulness. ${ }^{33,34}$

\section{Statistical analysis}

The Consolidated Standards of Reporting Trials (CONSORT) guidelines for randomised trials were followed, ${ }^{35}$ and the analyses were done using SPSS version 20 for Windows. Intention-to-treat (ITT) analyses were performed using the SPSS Missing Value Analysis to impute all missing data on the continuous measures with the expectation-maximisation method. This method estimates the unmeasured data based on maximum likelihood estimates using observed data on all continuous outcome measures in an iterative process. ${ }^{36}$ To provide a comprehensive picture of the effects of the intervention, the outcomes were analysed based on ITT as well as for those completing treatment only. The completers group was defined as participants who completed at least the first six sessions, as these sessions of the ACT intervention dealt with each of the six ACT processes. One-way analysis of variance (ANOVA) and chi-squared tests were conducted to examine baseline differences between the ACT intervention and the two control conditions. To examine the differences between the conditions on all the outcome measures, a 3 (group) $\times 3$ (time) repeated measures ANOVA was used. In the case of significant timegroup interactions, post hoc analysis of covariance (ANCOVA) was used on the change scores of the outcome measures with baseline scores as covariates. To examine the change from $T_{2}$ to $T_{3}$ in the two intervention groups, a 2 (group) $\times 2$ (time) repeated measures ANOVA and paired $t$-tests were used. Differences in non-study treatment (participants receiving other forms of care during the trial) were analysed using chi-squared tests on the proportions of non-study treatment between the three conditions.

Effect sizes at post-intervention were calculated with Cohen's $d$ using the means and the standard deviations of the measurements of the conditions. Cohen's $d$ is calculated as

$$
\frac{(\text { mean } 1-\text { mean } 2)}{\text { s.d. } \text { pooled }}
$$

where

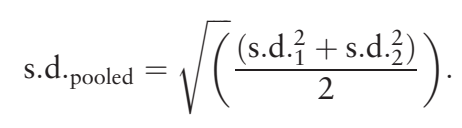

To interpret Cohen's $d$, effect sizes up to 0.49 were considered small, $0.50-0.79$ moderate, $0.80-1.29$ large and above 1.30 very large. ${ }^{37}$ For the changes within the groups $\left(T_{2} v . T_{3}\right)$ Cohen's $d$ was corrected for dependence among means by using the correlation between the two means. ${ }^{38}$ Comparisons were two-tailed and interpreted with a significance of $P<0.05$.

Using the Jacobson \& Truax method we determined the proportion of participants who made a clinically significant change on the CES-D from baseline to post-treatment. ${ }^{39}$ First, the reliable change was calculated with the reliable change index, calculated as

$$
\left(x_{2}-x_{1}\right) / S_{\text {diff }}
$$

where $x_{1}$ and $x_{2}$ are the individual's post-test and pre-test scores and $S_{\text {diff }}$ is the standard error of difference between the two test scores; $S_{\text {diff }}$ can be computed directly from the standard error of

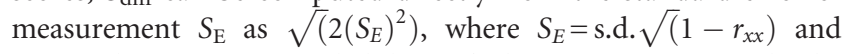
$r_{x x}$ is the test-retest reliability of the measure. Second, the recovery criterion was defined as a post-treatment score below the cut-off value of 16 for clinically relevant depressive symptoms. The score of 16 has been established in previous research as the cut-off indicating the presence of clinically relevant depressive symptoms. ${ }^{40,41}$ A clinically significant change on the CES-D is thus defined as a reliable change between the measurements and a post-treatment score below 16. Participants who had a clinically relevant change were coded 1 (implying favourable treatment response, 'success') or 0 ('failure'). The binary outcome was used to calculate the odds ratio (OR) using logistic regression. Based on the clinically significant change proportions, the number needed to treat (NNT) was calculated. ${ }^{42}$

In addition to the clinically significant change, the effect on diagnosis assessed with the MINI and the SDS was analysed using logistic regression. We calculated favourable treatment response when a participant was free from depression or did not develop 
a diagnosed depression at $T_{2}$ ('success'). This yields a binary outcome with failure coded as 0 and success as 1 . This binary outcome was then used to obtain odds ratios and the NNT. Differences between the proportions of change within the diagnosis group were analysed using chi-squared tests for comparisons between the two intervention conditions and the waiting-list control.

\section{Results}

A total of 436 persons were assessed for eligibility, of whom 93 were excluded based on initial screening criteria (Fig. 1). Owing to a computer error, data concerning 43 of those excluded were not stored. Reasons for exclusion based on data for the other 50 persons were insufficient time to participate $(34 \%, n=17)$, few

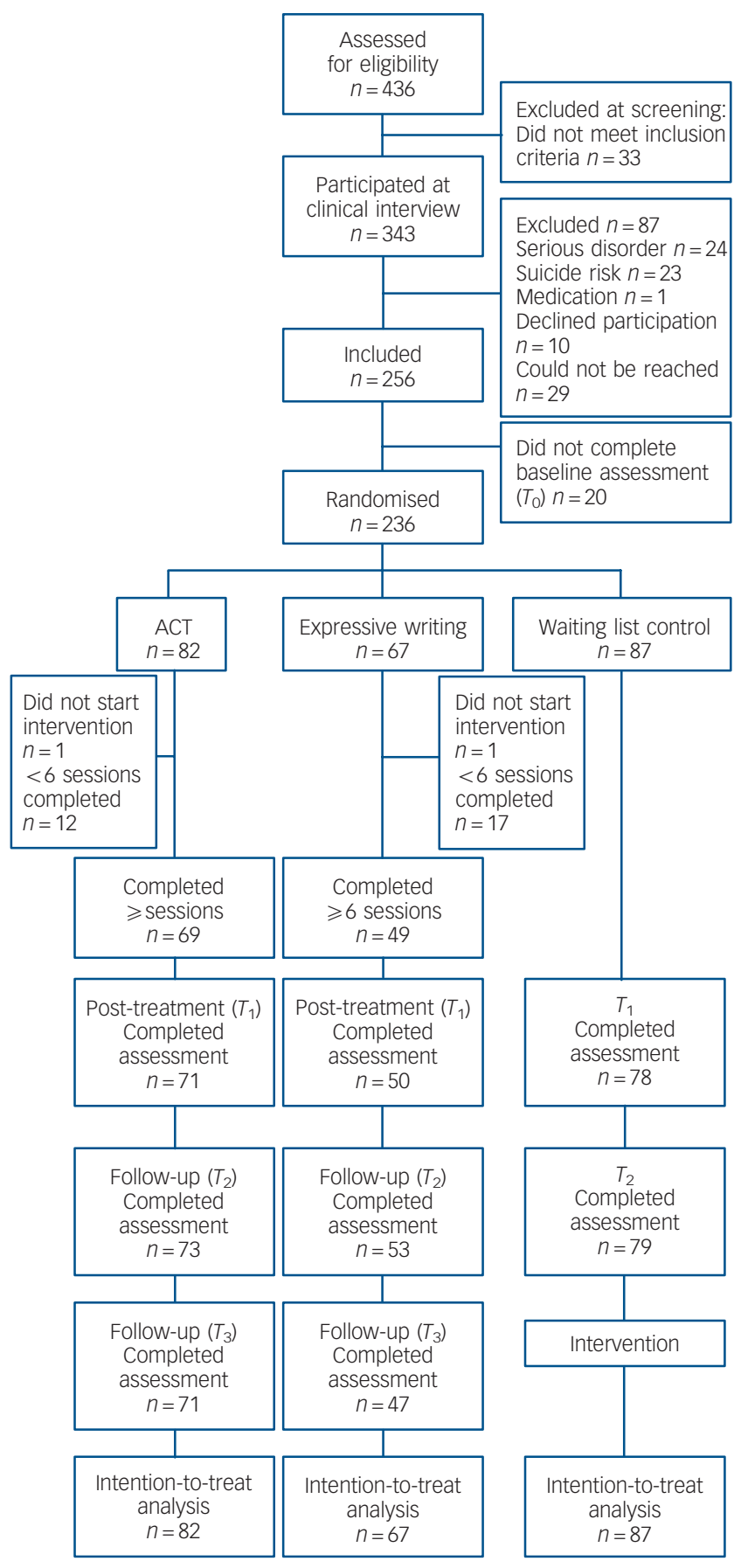

Fig. 1 Study profile. depression symptoms $(28 \%, n=14)$, current psychological or medication treatment $(20 \%, n=10)$, problems with the Dutch language $(16 \%, n=8)$ and age less than 18 years $(2 \%, n=1)$. A total of 343 participants met the initial screening criteria for inclusion and were contacted by telephone for a structured interview. On the basis of this interview 58 participants were excluded because of the seriousness of their disorder $(n=24)$, a high suicide risk $(n=23)$, current psychopharmacological treatment $(n=1)$ or refusal to participate $(n=10)$. A further 29 respondents could not be reached by telephone at the agreed time. Of the 256 included participants, 20 did not complete the baseline measurement. Hence a total of 236 participants were randomised.

After randomisation two participants (one in each intervention group) did not start the intervention for unknown reasons. Twelve participants in the ACT group did not complete the intervention (attended fewer than six sessions), compared with 17 in the expressive writing group. The main reasons given for non-completion were personal problems and that the intervention was too time-consuming. Adherence was $84 \%$ for the ACT group, compared with $76 \%$ for the expressive writing group. In the ACT group $73 \%$ of the participants completed all nine sessions, compared with $63 \%$ in the expressive writing group; the difference was not significant $\left(\chi^{2}(1, n=149)=1.87, P>0.05\right)$. Analyses of non-study treatment revealed no significant difference between the conditions: at $T_{1} \chi^{2}(2, n=175)=1.36, \quad P>0.05 ;$ at $T_{2}$ $\chi^{2}(2, n=202)=2.01, \quad P>0.05$. Treatment-completer analyses revealed similar results between completer and non-completer groups with regard to demographic variables and outcome measures. Also, per protocol analysis revealed similar outcomes. Therefore, only the results for the total sample on the imputed data are reported (with exception of the logistic regression of the diagnostic classification).

At $T_{1}$ (post-treatment for the intervention conditions) data were available for 199 participants (drop-out rates: ACT 13\%, expressive writing $25 \%$, waiting list $10 \%)$ and at $T_{2}$ (6-month follow-up for the intervention conditions and pre-intervention for the waiting-list condition) data were available for 205 participants (drop-out rates: ACT 11\%, expressive writing 21\%, waiting list 9\%). At $T_{3}$ (12-month follow-up for the intervention conditions) data were available for 118 of the 149 participants (drop-out rates: ACT 13\%, expressive writing 30\%).

\section{Baseline characteristics}

Table 1 gives an overview of the participants' characteristics. Eligible participants had a mean age of 46.8 years (s.d. $=12.1$, range $20-73)$, most were women (76\%), and two-thirds (66\%) had a high level of education. Mean baseline score on the CES-D was 26.73 (s.d. = 8.38). Of the 236 participants, $95(40 \%)$ were diagnosed with a mood disorder. Owing to a programming error in the randomisation procedure the number of participants in each condition differed. There was no significant difference at baseline between the conditions for any of the demographic variables or outcome measures, indicating a successful randomisation, except for gender: $\chi^{2}(2, n=236)=22.78, P<0.001$. A comparison of the results based on the analyses with $v$. without gender as a covariate revealed similar results. Therefore, only the results without gender as covariate are reported (additional analyses with gender as covariate are available from the authors on request).

\section{Outcomes}

Means and standard deviations for all outcome measures at baseline, post-treatment and follow-up and the results of the repeated measures are presented in Table 2. Figure 2 shows the 


\begin{tabular}{|c|c|c|c|c|}
\hline & $\begin{array}{c}\text { Total } \\
(n=236)\end{array}$ & $\begin{array}{c}\text { ACT } \\
(n=82)\end{array}$ & $\begin{array}{c}\text { EW } \\
(n=67)\end{array}$ & $\begin{array}{c}\text { WLC } \\
(n=87)\end{array}$ \\
\hline Age, years: mean (s.d.) ${ }^{a}$ & $46.85(12.06)$ & $45.15(10.78)$ & $46.73(12.65)$ & $48.54(12.63)$ \\
\hline Range & $20-73$ & $21-69$ & $20-69$ & $21-73$ \\
\hline \multicolumn{5}{|l|}{ Gender, $n(\%)^{\mathrm{b}}$} \\
\hline Female & $179(75.8)$ & $76(92.7)$ & $40(59.7)$ & $63(72.4)$ \\
\hline Male & $57(24.2)$ & $6(7.3)$ & $27(40.3)$ & $24(27.6)$ \\
\hline \multicolumn{5}{|l|}{ Marital status, $n(\%)^{a, c}$} \\
\hline Single & $81(35.5)$ & $28(36.4)$ & $21(32.8)$ & $32(36.8)$ \\
\hline Living with partner & $147(64.5)$ & $49(63.6)$ & $43(67.2)$ & $55(63.2)$ \\
\hline \multicolumn{5}{|l|}{ Nationality, $n(\%)^{\mathrm{a}, \mathrm{c}}$} \\
\hline Dutch & 204 (89.5) & $68(88.3)$ & $59(92.2)$ & 77 (88.5) \\
\hline Other & $24(10.5)$ & $9(11.7)$ & $5(7.8)$ & $10(11.5)$ \\
\hline \multicolumn{5}{|l|}{ Education level, $n$ (\%) } \\
\hline High $^{a}$ & $157(66.5)$ & $55(67.1)$ & $45(67.2)$ & $57(65.5)$ \\
\hline Middle $^{a}$ & 75 (31.8) & $24(29.3)$ & $22(32.8)$ & 29 (33.3) \\
\hline Low & $4(1.7)$ & $3(3.7)$ & $0(0.0)$ & $1(1.1)$ \\
\hline \multicolumn{5}{|l|}{ Daily activities, $n(\%)^{\mathrm{a}, \mathrm{c}}$} \\
\hline Paid job & $150(65.8)$ & $57(74.0)$ & $41(64.1)$ & $52(59.8)$ \\
\hline No job & 78 (34.2) & $20(26.0)$ & $23(35.9)$ & $35(40.2)$ \\
\hline \multicolumn{5}{|l|}{ Primary diagnosis, $n$ (\%) } \\
\hline No diagnosis ${ }^{a}$ & $97(41.1)$ & $36(43.9)$ & $22(32.8)$ & $39(44.8)$ \\
\hline Major depressive episode & $18(7.6)$ & $7(8.6)$ & $6(9.0)$ & $5(5.8)$ \\
\hline Recurrent depression ${ }^{a}$ & $61(25.9)$ & $21(25.6)$ & $21(31.3)$ & $19(21.8)$ \\
\hline Dysthymic disorder & $5(2.1)$ & $0(0.0)$ & $3(4.5)$ & $2(2.3)$ \\
\hline Other mood disorder & $11(4.7)$ & $2(2.4)$ & $4(6.0)$ & $5(5.8)$ \\
\hline Anxiety disorder ${ }^{a}$ & $44(18.6)$ & $16(19.5)$ & $11(16.4)$ & $17(19.5)$ \\
\hline \multicolumn{5}{|l|}{ Comorbidity, $n$ (\%) } \\
\hline Mood disorder ${ }^{a}$ & $54(22.9)$ & $22(26.8)$ & $17(25.4)$ & $15(17.2)$ \\
\hline Anxiety disorder & $3(1.3)$ & $0(0.0)$ & $3(4.5)$ & $0(0.0)$ \\
\hline History of diagnostic classification, $n(\%)^{a}$ & $55(23.3)$ & 19 (23.2) & $11(16.4)$ & $25(28.7)$ \\
\hline $\begin{array}{l}\text { ACT, acceptance and commitment therapy; EW, exp } \\
\text { a. No significant difference between intervention and } \\
\text { b. Significant difference between intervention and co } \\
\text { c. Owing to a computer error data on marital status, }\end{array}$ & $\begin{array}{l}\text { WLC, waiting-li } \\
\text { on }(P>0.05) \text {. } \\
(P<0.05) \text {. } \\
\text { daily activities }\end{array}$ & he $A C T$ & 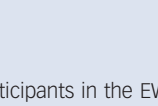 & akenly \\
\hline
\end{tabular}

repeated measure ANOVA for the primary outcome measure, the CES-D score. For all outcome measures significant interactions were found, except for the mindfulness facets observing $\left(F_{(2,233)}=2.38\right.$, $P=0.05)$ and acting with awareness $\left(F_{(2,233)}=1.16, P=0.33\right)$. Post hoc ANCOVA revealed that participants in the ACT intervention improved significantly more from $T_{0}$ to $T_{1}$ on all outcome measures compared with the waiting-list control group (all $P<0.01$ ), except for the mindfulness facet acting with awareness $(P=0.62)$. Compared with the expressive writing intervention post hoc ANCOVA revealed that participants in the ACT group improved significantly more from $T_{0}$ to $T_{1}$ on all outcome measures (all $P<0.05$ ), except for the mindfulness facets observing $(P=0.85)$, describing $(P=0.12)$ and acting with awareness $(P=0.63)$. No significant improvement was found from $T_{1}$ to $T_{2}$ for any of the conditions on any outcome measure.

Additional analyses from $T_{0}$ to $T_{2}$ showed that the ACT intervention group improved significantly more on all outcome measures compared with the waiting-list condition (all $P<0.05$ ) except for positive mental health $(P=0.06)$ and the mindfulness facets observing $(P=0.34)$ and acting with awareness $(P=0.06)$. This indicates that the ACT intervention shows a significant improvement over time compared with the waiting-list condition. The expressive writing intervention group only showed a significant improvement from baseline to follow-up compared with the waiting-list condition for psychological flexibility and the mindfulness facets describing, non-judging of inner experience and non-reactivity to inner experience (all $P<0.05$ ).

In Table 3 the effect sizes between the conditions at postintervention $\left(T_{1}\right)$ and 6-month follow-up $\left(T_{2}\right)$ are presented.
At $T_{1}$ moderate and small effect sizes were found on all the outcome measures for the ACT intervention compared with the waiting-list condition, and small effect sizes compared with the expressive writing intervention. For the latter intervention small effect sizes were found compared with the waiting-list condition. At $T_{2}$ small effect sizes were found on all the outcome measures for the ACT intervention compared with the other two

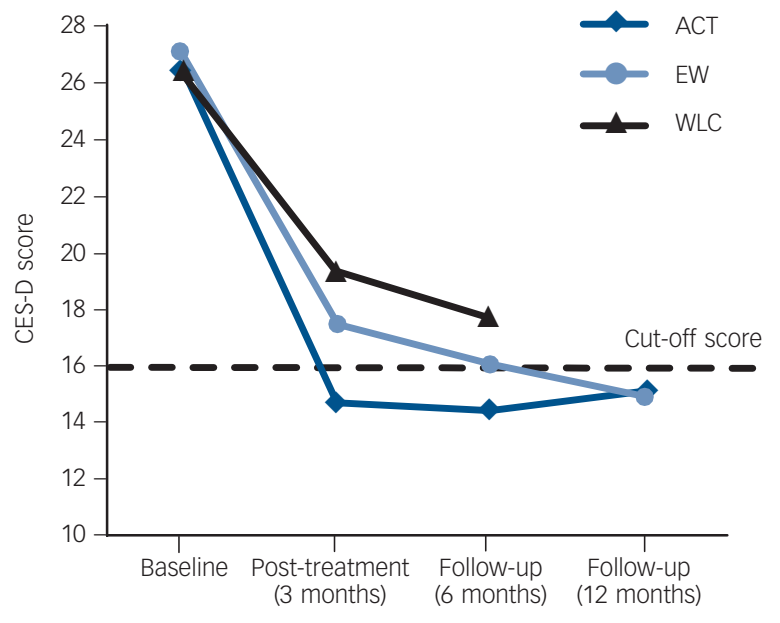

Fig. 2 Time $\times$ group repeated measures analysis of variance on Center for Epidemiologic Studies - Depression (CES-D) scores. ACT, acceptance and commitment therapy; EW, expressive writing; WLC, waiting-list control. 


\begin{tabular}{|c|c|c|c|c|c|}
\hline & \multicolumn{5}{|c|}{ score, mean (s.d.) } \\
\hline & Pre-treatment & Post-treatment & Follow-up 1 & Follow-up 2 & $F^{a}$ \\
\hline \multicolumn{6}{|l|}{ CES-D } \\
\hline ACT & $26.70(8.02)$ & $14.68(8.05)$ & $14.42(9.78)$ & $15.07(8.66)$ & \multirow[t]{3}{*}{$3.07^{*}$} \\
\hline EW & $27.07(9.17)$ & $17.51(7.87)$ & $16.06(8.78)$ & $14.96(9.59)$ & \\
\hline WLC & $26.51(8.16)$ & $19.34(8.55)$ & $17.71(10.72)$ & & \\
\hline \multicolumn{6}{|l|}{ HADS-A } \\
\hline ACT & $8.73(3.01)$ & $6.15(3.25)$ & $6.49(3.57)$ & $6.28(3.50)$ & \multirow{3}{*}{$3.50^{* *}$} \\
\hline EW & $9.20(2.86)$ & 7.47 (3.23) & $7.22(3.60)$ & 6.78 (3.44) & \\
\hline WLC & $8.70(2.99)$ & $7.82(3.62)$ & $7.45(3.76)$ & & \\
\hline \multicolumn{6}{|l|}{ MHC-SF } \\
\hline ACT & $2.47(0.76)$ & $3.03(0.89)$ & $3.05(0.95)$ & $3.13(0.94)$ & \multirow[t]{3}{*}{$2.70^{*}$} \\
\hline EW & $2.36(0.75)$ & $2.74(0.75)$ & $2.83(0.83)$ & $2.90(0.86)$ & \\
\hline WLC & $2.45(0.80)$ & $2.69(0.86)$ & $2.84(0.93)$ & & \\
\hline \multicolumn{6}{|l|}{ AAQ-II } \\
\hline ACT & $40.66(8.07)$ & $47.74(9.24)$ & $47.97(10.28)$ & $48.86(10.28)$ & \multirow{3}{*}{$4.95^{\star \star \star}$} \\
\hline EW & $38.93(6.88)$ & 44.15 (7.24) & $45.40(8.11)$ & $45.72(8.54)$ & \\
\hline WLC & $40.37(9.37)$ & $43.04(9.60)$ & $43.82(10.11)$ & & \\
\hline \multicolumn{6}{|c|}{ FFMQ-obs } \\
\hline ACT & $14.66(2.96)$ & $15.80(3.00)$ & $15.63(2.88)$ & $18.86(3.58)$ & \multirow[t]{3}{*}{2.38} \\
\hline EW & $14.14(2.83)$ & $15.37(2.54)$ & $15.37(2.38)$ & $18.30(2.87)$ & \\
\hline WLC & $14.61(3.18)$ & $14.99(3.10)$ & $15.33(3.01)$ & & \\
\hline \multicolumn{6}{|c|}{ FFMQ-des } \\
\hline ACT & $17.28(3.68)$ & 18.67 (3.33) & $18.51(3.80)$ & $15.73(3.00)$ & \multirow[t]{3}{*}{$3.76^{* *}$} \\
\hline EW & $16.81(3.46)$ & $17.69(3.47)$ & $18.16(3.25)$ & $15.21(2.47)$ & \\
\hline WLC & $17.70(3.22)$ & $17.69(3.85)$ & $17.84(3.90)$ & & \\
\hline \multicolumn{6}{|c|}{ FFMQ-act } \\
\hline ACT & $15.44(3.74)$ & $15.66(2.60)$ & $16.64(3.49)$ & 16.49 (3.59) & \multirow[t]{3}{*}{1.16} \\
\hline EW & $14.67(3.25)$ & $15.41(2.85)$ & $15.80(3.07)$ & $15.84(3.56)$ & \\
\hline WLC & $15.14(3.69)$ & $15.30(3.81)$ & $15.70(3.80)$ & & \\
\hline \multicolumn{6}{|l|}{ FFMQ-nj } \\
\hline ACT & $13.84(3.41)$ & $16.21(3.41)$ & $16.21(3.51)$ & $16.63(3.73)$ & \multirow[t]{3}{*}{$3.80 * *$} \\
\hline EW & $13.71(3.13)$ & $15.14(2.82)$ & $15.30(2.85)$ & $15.53(3.25)$ & \\
\hline WLC & $13.84(3.72)$ & $14.77(4.29)$ & $14.44(3.79)$ & & \\
\hline \multicolumn{6}{|l|}{ FFMQ-nr } \\
\hline ACT & $13.94(3.41)$ & $16.83(2.57)$ & $16.72(3.16)$ & $16.80(3.02)$ & \multirow[t]{3}{*}{$8.94^{* * *}$} \\
\hline EW & $14.37(3.06)$ & $15.90(2.59)$ & $15.98(2.55)$ & $16.15(2.65)$ & \\
\hline WLC & $14.38(2.78)$ & 14.69 (3.39) & $15.11(3.26)$ & & \\
\hline \multicolumn{6}{|c|}{$\begin{array}{l}\text { AAQ-II, Acceptance and Action Questionnaire II; ACT, acceptance and commitment therapy; CES-D, Center for Epidemiologic Studies - Depression scale; EW, expressive writing; } \\
\text { FFMQ, Five Facet Mindfulness Questionnaire (obs, observing; des, describing; act, acting with awareness; nj, non-judging of inner experience, nr, non-reactivity to inner experience), } \\
\text { HADS -A, Hospital Anxiety and Depression Scale - Anxiety subscale; MHC-SF, Mental Health Continuum - Short Form; WLC, waiting-list control. } \\
\text { a. Time } \times \text { group analysis of variance. }\end{array}$} \\
\hline
\end{tabular}

conditions. The expressive writing intervention also showed small effect sizes compared with the waiting-list condition.

\section{Effect maintenance}

Repeated measures ANOVA revealed no significant difference between the ACT and expressive writing interventions on the change in outcome measures from $T_{1}$ to $T_{3}$. Also, within-group analyses showed no significant change for both interventions on all outcome measures. Overall, both interventions showed maintenance of the effects on the primary and secondary measures from 6 months to 12 months of follow-up.

\section{Clinically significant change}

The reliable change on the CES-D turned out to be a pre-treatment to post-treatment difference of at least 7 scale points. The proportion of participants who reached a clinically significant change post-treatment on the CES-D in the ACT intervention was $54 \%(n=44)$ v. $26 \%(n=23)$ in the waiting-list control group $(\mathrm{OR}=3.22,95 \%$ CI $1.69-6.14, P<0.001 ; \mathrm{NNT}=3.7)$. In the expressive writing intervention $21(31 \%)$ reached a clinically significant change, resulting in a non-significant difference compared with the waiting-list control $(P=0.50)$. Comparison of the two interventions on clinically significant change posttreatment on the CES-D resulted in a significant difference in favour of ACT $(\mathrm{OR}=2.54,95 \%$ CI $1.29-4.98, P<0.01$; NNT $=4.5)$. At 6-month follow-up no significant difference was found for the proportion of participants who reached a clinically significant change on the CES-D in the ACT $(52 \%, n=43)$ and expressive writing interventions $(36 \%, n=24) v$. the waiting-list group $(44 \%, n=38)$. Comparison of the two interventions on clinically significant change at follow-up resulted in a significant difference in favour of ACT $(\mathrm{OR}=1.98,95 \%$ CI 1.02-3.83, $P<0.05$; NNT $=6.0)$.

\section{Effects on diagnostic classification}

Table 4 presents the results of the proportions of success for the MINI diagnoses $(n=204)$. Logistic regressions on the proportions of success were all non-significant, showing similar results of favourable outcome on the MINI. Chi-squared tests revealed significant proportional improvement in the diagnosis of recurrent depression for both ACT $\left(\chi^{2}(1, n=152)=6.03\right.$, 


\begin{tabular}{|c|c|c|c|c|c|c|}
\hline & \multicolumn{6}{|c|}{ Effect size } \\
\hline & \multicolumn{3}{|c|}{ Post-treatment } & \multicolumn{3}{|c|}{ 6-month follow-up } \\
\hline & ACT-WLC & ACT-EW & EW-WLC & ACT-WLC & ACT-EW & EW-WLC \\
\hline \multicolumn{7}{|c|}{ Primary outcome } \\
\hline CES-D & 0.56 & 0.36 & 0.22 & 0.32 & 0.18 & 0.17 \\
\hline \multicolumn{7}{|c|}{ Secondary outcomes } \\
\hline HADS-A & 0.49 & 0.41 & 0.10 & 0.26 & 0.20 & 0.06 \\
\hline MHC-SF & 0.39 & 0.35 & 0.06 & 0.22 & 0.25 & 0.01 \\
\hline AAQ-\| & 0.50 & 0.43 & 0.13 & 0.41 & 0.28 & 0.17 \\
\hline FFMQ-SF & 0.50 & 0.38 & 0.19 & 0.43 & 0.29 & 0.20 \\
\hline
\end{tabular}

\begin{tabular}{|c|c|c|c|c|c|c|}
\hline \multirow[b]{3}{*}{ Diagnosis } & \multicolumn{6}{|c|}{ Participants improved, $n$} \\
\hline & \multicolumn{2}{|c|}{$\mathrm{ACT}(n=72)$} & \multicolumn{2}{|c|}{ EW $(n=52)$} & \multicolumn{2}{|c|}{ WLC $(n=80)$} \\
\hline & $T_{0}$ & $T_{2}$ & $T_{0}$ & $T_{2}$ & $T_{0}$ & $T_{2}$ \\
\hline No diagnosis & 36 & 51 & 22 & 40 & 39 & 49 \\
\hline Major depressive episode & 7 & 2 & 6 & 0 & 5 & 1 \\
\hline Recurrent depression & 21 & 7 & 21 & 4 & 19 & 14 \\
\hline Dysthymic disorder & 0 & 1 & 3 & 0 & 2 & 0 \\
\hline Other mood disorder & 2 & 2 & 4 & 1 & 5 & 2 \\
\hline Anxiety disorder & 16 & 9 & 11 & 7 & 17 & 14 \\
\hline
\end{tabular}

$P<0.01)$ and the expressive writing intervention $\left(\chi^{2}(1, n=132)=15.9, P<0.001\right)$ compared with the waiting-list condition. Comparison of the two interventions on proportions of improvement in the diagnosis of recurrent depression was non-significant $\left(\chi^{2}(1, n=124)=2.83, P=0.09\right)$.

\section{Discussion}

This is the first study to investigate the effects of a web-based ACT intervention for adults with mild to moderate depressive symptoms in a randomised controlled trial. The effects of the intervention were compared with a waiting list and an active control condition and were measured at post-treatment and at 6-month and 12-month follow-up. Overall, the results show that in the short term the ACT intervention was significantly more effective on the primary outcome measure and most of the secondary outcome measures than both the waiting-list control and the expressive writing intervention, but that both interventions had similar effects on depressive symptoms and secondary measures after 6 months and 12 months.

In comparison with the waiting-list control, participants receiving ACT showed significantly larger reductions of depressive symptoms post-treatment. The effect size at that point was moderate and at the 6-month follow-up it was small. The likelihood of a clinically significant change in depressive symptoms at the post-treatment assessment was substantially higher in the ACT group, compared with both the waiting-list $(\mathrm{NNT}=3.7)$ and the expressive writing groups $(\mathrm{NNT}=4.5)$. Moreover, comparison of the expressive writing and waiting-list control groups resulted in a non-significant difference. This outcome suggests that ACT was superior to both the active intervention and the waiting-list control in directly decreasing clinically relevant depressive symptoms. This is relevant, as the presence of such symptoms is the most important risk factor for major depressive disorder and is associated with considerable economic costs. ${ }^{43}$ Moreover, the ACT intervention resulted in significantly larger post-treatment reductions in anxiety and improvements in positive mental health in comparison with the waiting-list condition. The effect sizes were in the small range at post-treatment and in the small (non-significant) range at 6-month follow-up.

\section{Interpretation of the findings}

The findings at post-treatment are in line with findings from other studies of web-based interventions for adult depression. ${ }^{6,7}$ The smaller effects at 6-month follow-up appear to be largely due to further recovery in the waiting-list condition. An explanation could be that the waiting-list group anticipated starting the intervention directly after filling in the questionnaire at follow-up. The maintenance of the effect in the ACT intervention is similar to findings in earlier studies about the effect of ACT on depression. ${ }^{12,13}$ Furthermore, this study shows that the effects of ACT were maintained at 12-month follow-up. Our results from the diagnostic classification analysis showed no significant difference in the proportions of success, meaning that in all conditions fewer participants were diagnosed with a mood disorder post-treatment. However, additional analyses showed a significant difference for both interventions on the diagnosis of recurrent depression compared with the waiting-list control. This finding suggests that both interventions may be especially effective for people with recurrent depression, but this needs to be interpreted with caution as our study was not powered for this. For the ACT intervention a possible explanation could be that mindfulness is a substantial component of the therapy. In all 
lessons participants are invited to meditate in order to increase awareness and acceptance of negative emotions and thoughts. It has been shown that meta-cognitive awareness, i.e. seeing thoughts as mental events that come and go, is an important process contributing to the prevention of the recurrence of depression in people with a history of depression. ${ }^{44}$ As the participants in the ACT intervention showed a larger increase in mindfulness in comparison with the waiting list condition, this could explain the differential effects on recurrent depression at 6-month follow-up. However, these results must be interpreted with care and need to be supported by more research.

\section{Effectiveness of expressive writing}

Although it was found that the ACT intervention had larger posttreatment effects for depressive symptoms than the expressive writing intervention, no difference was found at follow-up. This was contrary to our hypothesis, in which we expected ACT to have a larger treatment effect both post-treatment and at follow-up. One explanation could be that the attention and emotional support through email contact in both interventions contributed to the effects. Another explanation is that expressive writing is also an effective treatment. Expressive writing has often been applied as an intervention, in which people write about emotional events on at least 3 days a week for $15-30 \mathrm{~min}$ for 3 or 4 consecutive days. ${ }^{11}$ However, to make the format more equal to the ACT intervention the intervention was extended to 9 weeks. To enhance adherence to this longer intervention many components were added, one of which was psychoeducation on emotion regulation based on Gross's model. We found significant improvements from baseline to 6-month follow-up for the expressive writing group in psychological flexibility and the mindfulness facets describing, non-judging of inner experience and non-reactivity to inner experience compared with the waiting-list control. Writing about negative emotions for many weeks and writing about positive events may help people to regulate emotions in a way that is similar to ACT, i.e. diminishing avoidance and increasing acceptance of emotions. Some authors have suggested that emotional writing may yield larger effects, ${ }^{45,46}$ but there is no consensus on the mechanisms of change. Our findings suggest that a comprehensive expressive writing intervention could be an effective web-based intervention, but more research is needed to support this.

\section{Adherence}

Adherence to treatment was high, with only 13 out of $82(16 \%)$ participants in the ACT group attending fewer than six sessions and $73 \%$ completing all nine sessions. The web-based intervention was designed using the Center for eHealth Research and Disease Management road map to achieve a user-friendly application that fits the values of the stakeholders and to evaluate the process of development. ${ }^{22}$ The high level of adherence in our study may indicate feasibility and compatibility with the wishes of our participants. Furthermore, effort was made to include persuasive technology in the design of the web-based interventions, as persuasive design has been shown to be positively related to adherence. ${ }^{47}$

\section{Strengths and limitations}

A strength of our study is that the ACT intervention was compared not only with a waiting-list condition but also with an active control condition in the form of a web-based expressive writing intervention. Non-specific treatment factors such as attention and emotional support are generally recognised to be important for therapeutic effect. ${ }^{48}$ Also, inclusion criteria were kept broad to enhance generalisability with respect to the general population and the external validity of the study. Moreover, adding semi-structured interviews as diagnostic measures enhanced the reliability of the outcome measures. Finally, high attrition rates are common in studies of web-based interventions. ${ }^{49}$ In our study treatment adherence was as high as $84 \%$, in contrast to many previous internet studies that showed lower adherence rates. ${ }^{14}$

Some limitations also apply. The first and most important is that the recruitment strategy raises the possibility of self-selection bias (i.e. self-referral and motivation for time investment). This, and the fact that our participants tended to have high levels of education relative to the general public, raise questions on generalisability. It is a common finding in internet studies that highly educated women are especially prone to apply for guided web-based self-help interventions. ${ }^{49}$ Second, there was no assessment of interrater reliability of the diagnostic classification in this study. However, previous studies have provided justification for this method of assessing psychiatric disorders. ${ }^{50} \mathrm{~A}$ third limitation is the absence of competence measures of the counsellors because of the risk of not following the treatment protocol. However, all counsellors were supervised and the treatment protocol was highly standardised. Fourth, although we standardised the interventions to make them equivalent, the format of the interventions differed somewhat between treatments. The expressive writing intervention relied almost exclusively on textbased material, whereas the ACT used more experiential exercises (mindfulness exercises and interactive material) and relied more on a mix of text-based and picture-based material, which is in line with the theoretical underpinnings of the ACT model. ${ }^{8,9}$

\section{Study implications}

The clinical implications of this trial are that ACT can be effective as a web-based public mental health intervention for people with mild to moderate depressive symptoms, at least for women with medium to high levels of education. Although ACT was found to have larger effects than expressive writing in the short term, our findings also suggest that a comprehensive web-based expressive writing therapy might be a promising public mental health intervention. This needs to be corroborated in future studies.

\section{Wendy T. M. Pots, MSC, DClinPsych, University of Twente, Department of \\ Psychology, Health and Technology, Enschede, and Dimence, Community Mental Health Centre, Almelo; Martine Fledderus, PhD, University of Twente, Department of Psychology, Health and Technology, Enschede; Peter A. M. Meulenbeek, PhD, DClinPsych, University of Twente, Department of Psychology, Health and Technology, Enschede, and Community Mental Health Centre, Warnsveld; Peter M. ten \\ Klooster, PhD, Karlein M. G. Schreurs, PhD, Ernst T. Bohlmeijer, PhD, University Klooster, PhD, Karlein M. G. Schreurs, PhD, Ernst T. Bohlmeijer, PhD, Unive
of Twente, Department of Psychology, Health and Technology, Enschede, The Netherlands}

Correspondence: Wendy Pots, University of Twente, Department of Psychology, Health and Technology, Cubicus, Drienerlolaan 5, PO Box 217, 7500 AE Enschede, The Netherlands. Email: w.t.m.pots@utwente.nl

First received 7 Feb 2014, final revision 5 Dec 2014, accepted 6 Dec 2014

\section{Acknowledgements}

We acknowledge with many thanks the therapists and participants who took part in this study.

\section{References}

1 Munoz RF, Cuijpers P, Smit F, Barrera AZ, Leykin Y. Prevention of major depression. Annu Rev Clin Psychol 2010; 6: 181-212.

2 Bijl RV, Ravelli A, Van Zessen G. Prevalence of psychiatric disorder in the general population: results of the Netherlands Mental Health Survey and Incidence Study (NEMESIS). Soc Psychiatry Psychiatr Epidemiol 1998; 33 : 587-95. 
3 Cuijpers $\mathrm{P}$, Smit F. Subthreshold depression as a risk indicator for major depressive disorder: a systematic review of prospective studies. Acta Psychiatr Scand 2004; 109: 325-31.

4 Cuijpers P, Van Straten A, Warmerdam L, Van Rooy MJ. Recruiting participants for interventions to prevent the onset of depressive disorders: possible ways to increase participation rates. BMC Health Serv Res 2010; 10 181.

5 Andersson G, Cuijpers P. Pros and cons of online cognitive-behavioural therapy. Br J Psychiatry 2008; 193: 270-1.

6 Arnberg FK, Linton SJ, Hultcrantz M, Heintz E, Jonsson U. Internet-delivered psychological treatments for mood and anxiety disorders: a systematic review of their efficacy, safety, and cost-effectiveness. PLOS ONE 2014; 9: e98118.

7 Richards D, Richardson T. Computer-based psychological treatments for depression: a systematic review and meta-analysis. Clin Psychol Rev 2012; 32: $329-42$.

8 Hayes SC, Luoma JB, Bond FW, Masuda A, Lillis J. Acceptance and Commitment Therapy: model, processes and outcomes. Behav Res Ther 2006; 44: 1-25.

9 Hayes SC, Strosahl KD, Wilson KG. Acceptance and Commitment Therapy: The Process and Practice of Mindful Change. Guilford, 2011.

10 Cavanagh K, Strauss C, Forder L, Jones F. Can mindfulness and acceptance be learnt by self-help? A systematic review and meta-analysis of mindfulness and acceptance-based self-help interventions. Clin Psychol Rev 2014; 34: 118-29.

11 Pennebaker JW. Writing about emotional experiences as a therapeutic process. Psychol Sci 1997; 8: 162-6.

12 Fledderus M, Bohlmeijer ET, Pieterse ME, Schreurs KMG. Acceptance and commitment therapy as guided self-help for psychological distress and positive mental health: a randomized controlled trial. Psychol Med 2012; 42 485-95.

13 Bohlmeijer ET, Fledderus M, Rokx TAJJ, Pieterse ME. Efficacy of an early intervention based on acceptance and commitment therapy for adults with depressive symptomatology: evaluation in a randomized controlled trial. Behav Res Ther 2011; 49: 62-7.

14 Christensen $\mathrm{H}$, Griffiths KM, Farrer L. Adherence in internet interventions for anxiety and depression. J Med Internet Res 2009; 11: e13.

15 Sheehan DV, Lecrubier $Y$, Sheehan $\mathrm{KH}$, Amorim $\mathrm{P}$, Janavs J, Weiller $\mathrm{E}$, et al The Mini-International Neuropsychiatric Interview (M.I.N.I.): the development and validation of a structured diagnostic psychiatric interview for DSM-IV and ICD-10. J Clin Psychiatry 1998; 59: 22-33.

16 Van Vliet IM, De Beurs E. The MINI-International Neuropsychiatric Interview (M.I.N.I.). A brief structured diagnostic psychiatric interview for DSM-IV and ICD-10 psychiatric disorders [in Dutch]. Tijdschr Psychiatr 2007; 49: 393-7.

17 Leon AC, Olfson M, Portera L, Farber L, Sheehan DV. Assessing psychiatric impairment in primary care with the Sheehan Disability Scale. Int J Psychiatry Med 1997; 27: 93-105.

18 Bohlmeijer E, Hulsbergen M. A Beginner's Guide to Mindfulness: Live in the Moment. Open University Press, 2013.

19 Kashdan TB, Rottenberg J. Psychological flexibility as a fundamental aspect of health. Clin Psychol Rev 2010; 30: 865-78.

20 Aldao A, Nolen-Hoeksema S. Specificity of cognitive emotion regulation strategies: a transdiagnostic examination. Behav Res Ther 2010; 48: 974-83.

21 Kabat-Zinn J. Full Catastrophe Living: Using the Wisdom of Your Body and Mind to Face Stress, Pain and IIIness. Delacorte, 1990.

22 Kelders SM, Pots WT, Oskam MJ, Bohlmeijer ET, Van Gemert-Pijnen JE. Development of a web-based intervention for the indicated prevention of depression. BMC Med Inform Decis Mak 2013; 13: 1-11.

23 Frattaroli J. Experimental disclosure and its moderators: a meta-analysis. Psychol Bull 2006; 132: 823-65.

24 Gross JJ, Thompson RA. Emotion regulation: conceptual foundations. In Handbook of Emotion Regulation (ed. JJ Gross): 3-24. Guilford, 2007.

25 Radloff LS. The CES-D scale, a self report depression scale for research in the general population. Appl Psychol Meas 1977; 1: 385-401.

26 Haringsma R, Engels Gl, Beekman ATF, Spinhoven P. The criterion validity of the Center for Epidemiological Studies Depression Scale (CES-D) in a sample of self-referred elders with depressive symptomatology. Int J Geriatr Psychiatry 2004; 19: 558-63.
27 Spinhoven P, Ormel J, Sloekers PPA, Kempen GIJM, Speckens AEM, Van Hemert AM. A validation study of the hospital anxiety and depression scale (HADS) in different groups of Dutch subjects. Psychol Med 1997; 27: 363-70.

28 Zigmond AS, Snaith RP. The hospital anxiety and depression scale. Acta Psychiatr Scand 1983; 67: 361-70.

29 Lamers SM, Westerhof GJ, Bohlmeijer ET, Ten Klooster PM, Keyes CL. Evaluating the psychometric properties of the mental health Continuum-Short Form (MHC-SF). J Clin Psychol 2011; 67: 99-110.

30 Keyes CLM, Wissing M, Potgieter JP, Temane M, Kruger A, van Rooy S Evaluation of the mental health continuum-short form (MHC-SF) in Setswana-speaking South Africans. Clin Psych Psychother 2008; 15: 181-92.

31 Fledderus M, Oude Voshaar MAH, ten Klooster PM, Bohlmeijer ET. Further evaluation of the psychometric properties of the acceptance and action questionnaire-II. Psychol Assess 2012; 24: 925-36.

32 Bond FW, Hayes SC, Baer RA, Carpenter KM, Guenole N, Orcutt HK, et al. Preliminary psychometric properties of the Acceptance and Action Questionnaire-II: a revised measure of psychological inflexibility and experiential avoidance. Behav Ther 2011; 42: 676-88.

33 Baer RA, Smith GT, Hopkins J, Krietemeyer J, Toney L. Using self-report assessment methods to explore facets of mindfulness. Assessment 2006; 13 27-45.

34 Bohlmeijer E, Klooster PM, Fledderus M, Veehof M, Baer R. Psychometric properties of the five facet mindfulness questionnaire in depressed adults and development of a short form. Assessment 2011; 18: 308-20.

35 Moher D, Schulz KF, Altman DG. The CONSORT statement: revised recommendations for improving the quality of reports of parallel-group randomized trials. Ann Intern Med 2001; 134: 657-62.

36 Dempster AP, Laird NM, Rubin DB. Maximum likelihood from incomplete data via the EM algorithm. J R Stat Soc Series B Stat Methodol 1977; 39: 1-38.

37 Cohen JA. Power primer. Psychol Bull 1992; 112: 155-9.

38 Morris SB, DeShon RP. Combining effect size estimates in meta-analysis with repeated measures and independent-groups designs. Psychol Methods 2002; 7: $105-25$.

39 Jacobson NS, Truax P. Clinical significance: a statistical approach to defining meaningful change in psychotherapy research. J Consult Clin Psychol 1991; 59: 12-9.

40 Beekman ATF, Deeg DJH, Van Limbeek J, Braam AW, De Vries MZ, Van Tilburg W. Criterion validity of the Center for Epidemiologic Studies Depression scale (CES-D): results from a community-based sample of older subjects in the Netherlands. Psychol Med 1997; 27: 231-5.

41 Smit F, Ederveen A, Cuijpers P, Deeg D, Beekman A. Opportunities for costeffective prevention of late-life depression: an epidemiological approach. Arch Gen Psychiatry 2006; 63: 290-6.

42 Cook RJ, Sackett DL. The number needed to treat: a clinically useful measure of treatment effect. BMJ 1995; 310: 452-4.

43 Cuijpers P, Smit F, Oostenbrink J, De Graaf R, Ten Have M, Beekman A. Economic costs of minor depression: a population-based study. Acta Psychiatr Scand 2007; 115: 229-36.

44 Teasdale JD, Moore RG, Hayhurst H, Pope M, Williams S, Segal ZV. Metacognitive awareness and prevention of relapse in depression: empirical evidence. J Consult Clin Psychol 2002; 70: 275-87.

45 Niles AN, Haltom KEB, Mulvenna CM, Lieberman MD, Stanton AL. Randomized controlled trial of expressive writing for psychological and physical health: the moderating role of emotional expressivity. Anxiety Stress Coping 2014; 27: 1-17

46 Nazarian D, Smyth JM. An experimental test of instructional manipulations in expressive writing interventions: examining processes of change. J Soc Clin Psychol 2013; 32: 71-96.

47 Kelders SM, Kok RN, Ossebaard HC, Van Gemert-Pijnen JEWC. Persuasive system design does matter: a systematic review of adherence to web-based interventions. J Med Internet Res 2012; 14: e152.

48 Martin DJ, Garske JP, Davis MK. Relation of the therapeutic alliance with outcome and other variables: a meta-analytic review. J Consult Clin Psychol 2000; 68: 438-50.

49 Eysenbach G. The law of attrition. J Med Internet Res 2005; 7: e11.

50 Evans M, Kessler D, Lewis G, Peters TJ, Sharp D. Assessing mental health in primary care research using standardized scales: can it be carried out over the telephone? Psychol Med 2004; 34: 157-62. 